be refined in its usefulness and become integrated as part of everyday clinical practice, as major changes to clinical practice tend to be resisted by the medical profession.

Ravi Sohal Staff Grade Psychiatrist, Bowden House Clinic Psychiatric Intensive Care Unit, London Road, Harrow on the Hill, Middlesex HA1 3JL

\section{Physical health of patients in rehabilitation and recovery}

Dr Greening's survey of the attention paid to physical health parameters of patients in rehabilitation and recovery (Psychiatric Bulletin, June 2005, 29, 210-212) highlighted inadequacies in routine monitoring and a lack of clear guidelines from policymakers over what a full assessment might constitute (National Collaborating Centre for Mental Health, 2003). Standards of competence in physical examination among psychiatric trainees have been widely denigrated and suggestions have been made regarding expected practice (Garden, 2005).

In January 2005 we audited standards of physical healthcare on an acute psychiatric unit and found wide variations in the use of routine blood tests, urinalysis and body mass index (BMI) monitoring With increasing attention paid to the metabolic effects of schizophrenia and of atypical anti-psychotics (Jin et al, 2004) it was felt that clinicians needed to agree a minimum standard for routine testing of all in-patients. Following discussion of this audit at the unit's academic meeting, views were assembled over which tests should be regarded as routine. An investigations summary sheet was designed, similar to those used on medical units, and included BMI and prolactin. This allows changes over time to be tracked at a glance and has been added to the admission notes to prompt requests for appropriate tests. The aim is to re-audit these standards in 1 year in anticipation that they will have translated into a more rigorous approach to the physical health of psychiatric in-patients.

GARDEN, G. (2005) Physical examination in psychiatric practice. Advances in Psychiatric Treatment, 11, 142-149.

JIN, H., MEYER, J. M. \& JESTE, D. V. (2004) Atypical antipsychotics and glucose dysregulation: a systematic review. Schizophrenia Research, 71 195-212.

NATIONAL COLLABORATING CENTRE FOR MENTAL HEALTH (2003) Schizophrenia: Full National Clinical Guideline on Core Interventions in Primary and Secondary Care. London and Leicester: Gaskell and the British Psychological Society.

Alexandra Pitman Senior House Officer in Psychiatry, *Michael Phelan Consultant Psychiatrist, Hammersmith and Fulham Menta Health Unit, Charing Cross Hospital, LondonW6 8NF 